\title{
Preliminary Study of Forward-Backward Time-Stepping Technique with Edge-Preserving Regularization for Object Detection Applications
}

\author{
Yong Guang $^{\# 1}$, Kismet Anak Hong Ping ${ }^{\sharp 2}$, Andrew Sia Chew Chie ${ }^{\# 3}$ \\ $\mathrm{Ng}$ Shi Wei ${ }^{\# 4}$, Thelaha Masri ${ }^{\# 5}$ \\ "Department of Electrical \& Electronic Engineering, Faculty of Engineering, \\ Universiti Malaysia Sarawak, 94300 Kota Samarahan, Sarawak, Malaysia \\ 13010042@siswa.unimas.my, ${ }^{2}$ hpkismet@ feng.unimas.my, ${ }^{3}$ 13020042@siswa.unimas.my \\ ${ }^{4} 13010044 @$ siswa.unimas.my, ${ }^{5}$ mthelaha@feng.unimas.my
}

\begin{abstract}
Forward-Backward Time-Stepping (FBTS) technique is used for the detection, imaging and reconstruction of an embedded object which is formulated at the time-domain utilizing Finite-Difference Time-Domain (FDTD) method. In order to solve FBTS inverse scattering problem, edge-preserving regularization is integrated. Image reconstruction results illustrated that the FBTS integrated with an edge-preserving regularization technique has the potential to detect the presence of the embedded object accurately. In this paper, an extended algorithm is shown in time-domain image reconstruction.
\end{abstract}

Keywords-Inverse scattering; Edge-Preserving regularization technique; Image reconstruction; Object detection

\section{INTRODUCTION}

Inverse scattering in the microwave imaging has received attention by many researchers for the past years. The research has been focused on a variety of industrial, military, medical application and geophysical [1-4]. The reason for this is because microwave imaging has enormous advantages to infer the characteristic of embedded objects in a given medium illuminated by electromagnetic waves. This technique can directly provide the distribution of the dielectric properties, shape and also the location of the embedded object. Besides, this technique is less expensive compared to the some wellknown technique such as the computed tomography and positron emission tomography. Furthermore, it is non-invasive and safer with low-level nonionizing radiation compared to the X-ray mammography. Therefore, it has the biggest potential to detect the buried objects, non-destructive examination, investigate the materials electromagnetic properties, and non-invasive medical diagnostics [5-9].

The goal of the microwave imaging is to make use of the scattered data received by the antennas to reconstruct the information (shape, location, and dielectric properties) of the embedded object. Since the microwave imaging is a non-linear inverse scattering problem, it must be solved and regularization is needed to avoid the ill-posed nature $[10,11]$. This inverse scattering could be coped with either frequencydomain or time-domain. However, time-domain has been revealed that it has the potential to reconstruct the dielectric properties more accurately [4, 12-15]. Currently, several time- domain inverse scattering methods have been considered, including Lagrange multipliers technique by Rekanos [14], the time-domain inverse scattering by Winters et al. [15] and the FBTS method by Takenaka et al. [4, 12, 13].

In this paper, the 2-D FBTS algorithm extended with the integration of the edge-preserving regularization technique. The edge-preserving regularization technique is aimed to solve the ill-posed nature occur in the above FBTS method. Thus, the combination of the FBTS algorithm and edge-preserving regularization technique can enhance the reconstruction in terms of convergence and stability of the optimization technique. Preliminary study of the simple object is explored to determine the efficiency of the combination for both schemes.

\section{FORWARD-BACKWARD TIME-STEPPING METHOD}

The target of the electromagnetic inverse scattering problem is to determine the object's shape, location and electrical properties (e.g. permittivity, $\varepsilon$ and electric conductivity, $\sigma$ ).

Fig. 1 shows a typical configuration of an active microwave tomography setup for FBTS inverse scattering in a two-dimensional field with an unknown object is placed in a free space.

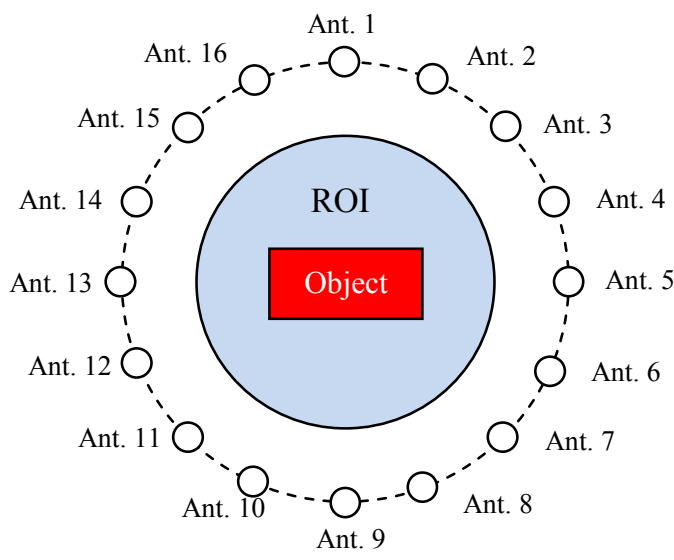

Fig. 1 Configuration of the FBTS technique in 2-D view 